\title{
Great need for changes in higher education in Greece
}

Bakoyiannis, Ioannis ; Cherdyntseva, Veroniki ; et al

DOI: https://doi.org/10.1515/cclm-2017-0188

Posted at the Zurich Open Repository and Archive, University of Zurich ZORA URL: https://doi.org/10.5167/uzh-207529

Journal Article

Published Version

Originally published at:

Bakoyiannis, Ioannis; Cherdyntseva, Veroniki; et al (2017). Great need for changes in higher education in Greece. Clinical Chemistry and Laboratory Medicine, 55(10):e238-e239.

DOI: https://doi.org/10.1515/cclm-2017-0188 


\section{Letter to the Editor}

Ioannis Bakoyiannis*, Veroniki Cherdyntseva*, Maria Aivalioti, Maria Barton, Davide Coda, Ioanna Douka, Angelina Evangelou, Christos Evangelou, Petros Ioannou, Vasiliki Kanta, Konstantinos Kapanidis, Ioannis Karagiannidis, Damianos Kokkinidis, Sofia Kouni, Jamie Lehnen, Andreas Patsalos, Julian Pei, Peristera-loanna Petropoulou, William Rodemer, Diego Scerbo, Alketa Stefa, Elizar Tsvetkov, Lazaros Vasilikos, Themistoklis Vasilopoulos, Aleksandra Vukojicic and Efthalia Zafeiropoulou

\section{Great need for changes in higher education in Greece}

DOI 10.1515/cclm-2017-0188

Received March 3, 2017; accepted March 7, 2017; previously published online April 20, 2017

Keywords: economic; education; international education; political.

To the Editor,

Over the past years, Greece has been witnessing a variety of social, economic and political upheavals. Due to such difficulties, the number of people seeking for a better quality of life abroad is continuously rising. Nowadays, the phenomenon of "brain drain", which describes the migration of young and aspiring scientists towards such countries, is very much common.

Numerous factors have fortified these results. First of all, research was importantly eliminated during the economic crisis. Funding sources were reduced and research grants decreased to less than $50 \%$. Research centers lack national funding, which compromises availability of

*Corresponding authors: Ioannis Bakoyiannis, Dublin City University, Dublin, Ireland; and National and Kapodistrian University of Athens, Laboratory of Experimental Surgery and Surgical Research "N.S. Christeas," Medical School, Athens, Greece, E-mail: johnbakneuro@gmail.com; and Veroniki Cherdyntseva, University of Athens, Athens, Greece, E-mail: ch.veronica@yahoo.com Maria Aivalioti: Albert Einstein College of Medicine, Bronx, NY, USA Maria Barton and William Rodemer: Temple University, Philadelphia, PA, USA

Davide Coda: Francis Crick Institute, London, UK

Ioanna Douka: University of Patras, Panepistimioupoli Patron, Greece Angelina Evangelou, Vasiliki Kanta, Alketa Stefa and Themistoklis Vasilopoulos: Rutgers University, Newark, NJ, USA Christos Evangelou: University of Manchester, Manchester, UK Petros loannou: University Hospital of Heraklion, University of Crete, Crete, Greece materials and equipment. The majority of graduate students have lost their stipend coverage and do not have the chance to acquire one. Such burdens restrain and compromise academic and research excellence. Therefore, the apparent choices for the new scientists are either to change occupation or leave the country in order to find a promising position of their interest abroad. This is definitely not the future young and ambitious students from around the world dream about when they make the decision to enter the world of academia and scientific research.

The number of Greek scientists that had successfully completed their studies in the field of their interest in Greece and are currently working abroad is vast. Some of them have pursued successful academic careers and their work already constitutes a great contribution to the societies they live in. Does not Greece deserve such a skilled and passionate workforce? It seems that the Greek society is inadequately aware of the importance of science in life. Greek universities score quite low in global ranking studies compared to other universities of the European Union.

Konstantinos Kapanidis: Aristotelian University of Thessaloniki, Thessaloniki, Greece

loannis Karagiannidis: Städtisches Klinikum Dessau, Auenweg, Germany

Damianos Kokkinidis: Denver VAMC, University of Colorado, Aurora, CO, USA

Sofia Kouni: University of Athens, Athens, Greece Jamie Lehnen: University of Houston, Houston, TX, USA Andreas Patsalos: University of Debrecen, Debrecen, Hungary Julian Pei: University of Pennsylvania, Philadelphia, PA, USA Peristera-Ioanna Petropoulou, Diego Scerbo and Aleksandra Vukojicic: Columbia University, New York, NY, USA Elizar Tsvetkov: Medical University-Plovdiv, Plovdiv, Bulgaria Lazaros Vasilikos: University of Zurich, Zurich, Switzerland Efthalia Zafeiropoulou: University of Paul-Valéry, Montpellier III, France 
It is also a fact that non-public universities are not allowed to be founded in Greece by law. Even the concept of private or non-public university is insufficiently understood in Greece. On the other hand, public/state universities, despite the good quality of many students and some of the faculty, deal with unprecedented corruption that compromises meritocracy and excellence.

We, students from Greek and international universities, envision a university system without the involvement of any political parties in the operation of universities that makes things more complicated. There is clearly another way of student groups in colleges we dream of. This includes psychological, foreign language, sports, book reading and other societies and clubs that give students the opportunity to enrich their knowledge and self-improve.

Greece is now in the process of constitutional amendment, which constitutes an important opportunity for Greece to make some major changes. Various high-rank universities established abroad are not national/public. The combination of state and non-public non-profit institutions can lead to more academic opportunities. This occurs for centuries in other countries in Europe, including the UK, which attracts the best of the young people from around the world. However, in Greece, this is surprisingly not yet allowed by the constitution. It is certain that new universities would boost the quality of education and research in Greece and would attract international students due to low costs of both fees and life. Considering that countries such as Germany and countries of Scandinavia have already combined programs in both the mother language and English, it is time for Greece to join in, compete and collaborate with them. This would definitely motivate researchers of Greek descent, who live abroad, or non-Greek researchers to relocate to Greece. This will create an excellent research environment that will include researchers from both public and non-public universities in Greece, constituting a "reservoir" of a powerful national research workforce. This can definitely be a way of reversing the "brain drain" that Greece is currently confronting, and stimulate the economy via discoveries that will lead to new products and applications, of which Greek universities will hold the intellectual property rights.

As Hippocrates has said "Healing is a matter of time, but it is sometimes also a matter of opportunity". This is the opportunity for Greece to catch up with modern developments in higher education and research.

Author contributions: All the authors contributed equally to this work, and have accepted responsibility for the entire content of this submitted manuscript and approved submission.

Research funding: None declared. Employment or leadership: None declared. Honorarium: None declared.

Competing interests: The funding organization(s) played no role in the study design; in the collection, analysis, and interpretation of data; in the writing of the report; or in the decision to submit the report for publication. 